\title{
Las Juntas de Gobierno en el contexto del derecho a la educación superior. La experiencia de la Universidad Veracruzana en el proceso de designación rectoral 2017
}

Jaqueline-del-Carmen Jongitud-Zamora y Claudio-Rafael Castro-López

\section{RESUMEN}

Se analiza el modelo de designación de rector(a) por juntas de gobierno, desde el enfoque de derechos humanos, y se describe una serie de innovaciones en el proceso de designación rectoral 2017 de la Universidad Veracruzana, con el objetivo de mostrar un conjunto de acciones y estrategias que inciden de forma positiva en el ejercicio de la atribución concedida a las juntas de gobierno, en el marco del derecho a la educación superior. Asimismo, en el texto se identifican áreas de oportunidad para la mejora en la realización de los procesos de designación por parte de estos órganos colegiados.

Palabras clave: comunidad universitaria, democratización, derechos humanos, gobierno universitario, instituciones de educación superior, México. 


\section{As Juntas de Governo no contexto do direito à educação superior. A experiência da Universidade Veracruzana no processo de designação reitoral 2017}

\section{RESUMO}

Se analisa o modelo de designação de reitor(a) por juntas de governo, partindo do enfoque de diretos humanos, e se descreve uma serie de inovações no processo de designação reitoral 2017 da Universidade Veracruzana, com o objetivo de mostrar um conjunto de ações e estratégias que incidem de forma positiva no exercício da atribuição concedida às juntas de governo, no marco do direto à educação superior. Também, se identificam no texto espaços oportunos para a melhora na realização dos processos de designação por parte destes órgãos colegiados.

Palavras chave: comunidade universitária, democratização, diretos humanos, governo universitário, instituições de educação superior, México.

\section{The Boards of Government in the Context of the Right to Higher Education. The Experience of the Universidad Veracruzana in the Process of Designation of the Rector in 2017}

\section{ABSTRACT}

This article analyzes the model of designation of the vice-chancellor rector by government boards, from a human rights perspective, and describes a series of innovations in the process of designation of this position in 2017 of the University of Veracruz, with the aim of showing a set of actions and strategies that positively affect the exercise of the attribution granted to government boards, within the framework of the right to higher education. This study also identifies areas of opportunity for improvement in the implementation of the appointment processes by these collegial groups.

Key words: university community, democratization, human rights, university government, higher education institutions, Mexico. 


\section{La Junta de Gobierno como modelo de gobierno universitario}

Como lo ha documentado Imanol Ordorika (2015), las formas de selección de las máximas autoridades universitarias, en particular de los rectores, ${ }^{1}$ muestran diferencias significativas en México y el mundo, lo que cabe relacionar con las trayectorias históricas institucionales y con la evolución de los sistemas políticos particulares en los cuales las instituciones de educación superior (IES) se encuentran inscritas (López, 2014; Ordorika, 2015).

Pese a las diferencias entre las formas de selección, la doctrina especializada ha logrado identificar cuatro modelos básicos (López, 2010; López, González, Mendoza y Pérez, 201 la; López et al., 2011b, López, 2013; Ordorika, Martínez y Ramírez, 2011; Ordorika, 2014, 2015), atendiendo a los procedimientos empleados y al nivel de participación de los grupos que integran a la comunidad universitaria. Dichos modelos o métodos de selección son: 1. Elección por voto directo; 2. Elección por órgano colegiadorepresentativo (consejos o asambleas universitarias); 3. Designación por juntas de gobierno o directivas, y 4. Nombramiento por órgano externo (gubernamental y/o empresarial). Cada uno de ellos presenta ventajas y desventajas y cuenta con defensores y detractores.

Todos los modelos de selección están presentes en México (Ordorika, 2015) y han sido asociados, documentados y estudiados empíricamente respecto a casos concretos de IES públicas del país (López et al., 2011 a; López, 2013). Sin embargo, son los métodos de selección por órgano colegiado-representativo y por juntas de gobierno los que predominan, siendo utilizados, en 2015, el primero por 16 IES públicas y el segundo por 15; mientras que el modelo de selección por voto directo es adoptado en siete institucio$n^{2}{ }^{2}$ y el nombramiento por órgano externo sólo en cuatro: la Universidad Nacional Abierta y a Distancia de México, la Universidad Pedagógica Nacional, el Instituto Politécnico Nacional y el Tecnológico Nacional de México, cuyos rectores son designados por el secretario de Educación Pública, en términos de los artículos 46 y 4, fracción XXI, del Reglamento Interior de la Secretaría de Educación Pública (Gómez, 2015b; López et al., 201 la; Ordorika, 2015).

Como se señaló anteriormente, cada uno de los modelos de selección presenta ventajas y desventajas, siendo posible cuestionarlos a partir de diversas perspectivas teóricas en relación no sólo con su diseño (lo formal), sino también con los efectos de su implementación (lo real). En este estudio el interés se centra en el modelo de selección por juntas de gobierno, y en particular en una de sus expresiones, la de la Universidad Veracruzana (UV), de ahí que en seguida sólo se aborde la caracterización general de este modelo en relación con el caso de la UV y se describa cómo ha sido valorado por los especialistas en el tema, remitiendo a quienes desean profundizar en los restantes métodos de selección a la literatura hasta ahora citada.

En el modelo de selección sujeto a estudio, la elección del rector(a) recae en un pequeño grupo de personas de reconocido prestigio y de relevante trayectoria (López et al., 201 la) o, en términos de la Ley de Autonomía de la Universidad Veracruzana, de personas honorables, de reconocido prestigio y destacadas en su especialidad, en la comunidad académica estatal o nacional (artículo 6, fracciones II, III y IV).

En México, los integrantes de las juntas de gobierno, en casi todas las IES públicas, son electos por los consejos universitarios (López et al., 201 la), hipótesis que incluye el caso de la UV pues los miembros de su Junta son electos por el Consejo Universitario General de una terna propuesta por la misma, esto en

\footnotetext{
${ }^{1}$ En otros contextos denominados también directores, presidentes o cancilleres (Ordorika, 2015).

${ }^{2}$ A saber, las universidades autónomas de Baja California Sur, Benito Juárez de Oaxaca, Chapingo, Goahuila, Guerrero y Zacatecas, así como la Autónoma Agraria Antonio Narro.
} 
términos de la fracción VI del artículo 4 de la Ley de Autonomía. En sentido contrario a la tendencia nacional (López et al., 2011a), la Junta de Gobierno de la UV tiene una composición que integra tanto a miembros internos, que forman parte de la institución, como miembros externos, pertenecientes a otras IES del país. De acuerdo con el artículo 3 del Reglamento de la Junta de Gobierno (RJG) los miembros internos son cinco y los externos cuatro.

A decir de Romualdo López et al. (2011a), todas las juntas de gobierno de las IES públicas de México llevan a cabo auscultaciones a la comunidad universitaria para conocer su opinión sobre los candidatos(as) a rector(a), entrevistan a los candidatos y deben tomar su decisión por mayoría calificada. Aspectos todos que se determinan, en el caso de la UV, en los artículos 8 y 9 de su Ley de Autonomía y del 19 al 27 del RJG. A todo esto vale la pena agregar que para ser aspirante a la rectoría de la UV se debe ser propuesto por algún miembro de la comunidad universitaria (art. 20, fracción II, 21 y 22 del RJG) y que la Junta de esta IES tiene la facultad de solicitar a los candidatos su biografía académica, con documentación comprobatoria, y su propuesta de programa de trabajo (art. 25 del RJG), además de estar sujeta a criterios normativos para establecer un juicio de idoneidad al momento de adoptar la decisión sobre quién ha de ser designado como rector(a) de la institución (art. 28 del RJG) y de tener la obligación de fundamentar y motivar su decisión (art. 32 RJG).

Por otra parte, los aspectos que se destacan como positivos del modelo de selección por juntas de gobierno son, en general, que se trata de un proceso mucho más fluido, menos problemático, que ha permitido una mayor estabilidad y continuidad en el desarrollo institucional de las IES públicas autónomas del país, que coadyuva con la toma de decisiones más académicas que políticas, sin prescindir de la participación de la comunidad universitaria, como en el método de nombramiento del rector(a) por un órgano externo, y que constituye una alternativa al modelo de voto directo, al ser las IES organizaciones sociales peculiares, dedicadas al cultivo del conocimiento (Acosta, 2014; López et al., 201 1a; López, 2013).

En contrapartida, las críticas al modelo enfatizan el carácter autoritario y secreto del nombramiento del rector(a). Asimismo, apuntan al enquistamiento de grupos de interés en el seno de las universidades, a la subordinación de las juntas al poder ejecutivo federal o local, según corresponda (Ordorika et al., 2011; Ordorika, 2015; Vargas y Olivares, 2007), a la disminución o pérdida del dinamismo en la participación de la comunidad universitaria, al secuestro de facto de las juntas de gobierno por parte de grupos de interés internos y/o externos y, a veces, a la falta de honorabilidad de sus integrantes; todo lo cual ha desembocado en que, en ocasiones, las decisiones de estos órganos sean ampliamente cuestionadas por diversos sectores de la comunidad universitaria involucrada (Abello, 2015; López, 2013; Ramírez, 2013).

La literatura explorada hasta el momento ha abordado al gobierno universitario (Casanova y Rodríguez, 2014) desde la tradición de las ciencias sociales, en particular desde la pedagogía, la historiografía universitaria, la sociología o la filosofía de la educación, así como desde la perspectiva de la gestión de las IES. En este estudio, el enfoque asumido es jurídico, en específico el que emerge normativa y doctrinalmente del campo de estudio de los derechos humanos (DH). En tal sentido, aporta una visión del modelo de selección del rector(a) por juntas de gobierno desde una perspectiva que aún no ha sido reportada por la literatura especializada y que permite identificar en qué medida dicho método garantiza el derecho fundamental en torno al cual gravita, esto es, el derecho humano a la educación superior o a la enseñanza superior, como también es denominado.

\section{Las juntas de gobierno en el marco del derecho humano a la educación superior}

La educación superior es un derecho humano, pues como tal ha sido reconocida en tratados universales 
y regionales de DH (Contreras et al., 2013), porque tal status le ha sido reiterado por órganos autorizados para la interpretación de tales acuerdos internacionales y porque los Estados han continuado con el desarrollo de normativa internacional en torno a él y con la incorporación, paulatina, a su derecho interno (Jongitud, 2017).

La ambigüedad en México sobre el carácter de derecho humano de la educación superior, fue superada con la reforma constitucional de 2011, en materia de DH (Jongitud, 2014). De ello empiezan a dar cuenta de forma consistente los criterios generados por la Suprema Corte de Justicia de la Nación (SCJN), en su décima época. ${ }^{3}$ Así, la Corte ha analizado el contenido, características y fines del derecho a la educación superior (SCJN, 2017a) y ha sentado jurisprudencia en el sentido de que el Estado tiene la obligación de implantar de forma progresiva su gratuidad (SCJN, 2017b). Asimismo, tribunales federales del país han empezado a generar criterios en torno a la garantía de este derecho, tal es el caso de la tesis de tribunales colegiados que apunta a que ciertos actos de universidades privadas pueden ser impugnados a través del amparo indirecto (TCC, 2017a, 2017b).

De conformidad con el artículo $3^{\circ}$, fracción VII, de la Constitución Federal, las IES autónomas tienen la facultad y la responsabilidad de gobernarse. Esta norma entraña, en relación con el tema que aquí interesa, dos facetas. Por un lado, el poder reconocido a las IES autónomas de decidir cuáles serán sus órganos de gobierno, de determinar la forma de seleccionar a sus autoridades y de elegir a las mismas de entre sus miembros. ${ }^{4}$ Por otro, la obligación de responder por el uso indebido que pudiesen hacer del poder que les ha sido encomendado.

En el anterior orden de ideas, las universidades autónomas son libres de elegir la forma o el método de selección del rector(a), cualesquiera que sea, pero dicha potestad debe ejercerse de tal forma que no implique restricción alguna al derecho a la educación superior (ES) y que, a la vez, coadyuve con su más efectiva realización.

El derecho a la ES, como otros que se integran en la categoría de DH, exige a todas las autoridades del país, en sus respectivos ámbitos de competencia, cumplir con obligaciones generales, ${ }^{5}$ elementos esenciales $^{6}$ y principios de interpretación y aplicación (Contreras et al., 2013). ${ }^{7}$ En tal sentido, y dado que el ejercicio de las atribuciones de las IES autónomas se dirige a satisfacer tal derecho, deben asegurarse de que todas sus acciones, incluidos los métodos y procedimientos empleados para la selección de sus autoridades, satisfagan tales criterios. En relación con esto, vale la pena destacar que según tesis de la Primera Sala de la SCJN (2016) "La autonomía universitaria, en tanto garantía institucional de un derecho humano [...] está subordinada a la maximización de éste, por lo que, por regla general, el ejercicio legítimo de la autonomía universitaria no puede incluir la restricción de aspecto alguno del derecho a la educación".

Así las cosas, del cúmulo de elementos que cabe considerar en materia de DH, interesa aquí destacar sobre todo los principios transversales de

\footnotetext{
${ }^{3}$ Las épocas son las etapas en las que se agrupan los criterios de los tribunales federales del país. La décima época dio inicio el 4 de octubre de 2011 en virtud de las reformas en materia de amparo y de DH.

${ }^{4}$ Como puede observarse, el modelo de designación del rector(a) por un órgano externo no corresponde al régimen de autonomía establecido en la fracción VII del artículo $3^{\circ}$ constitucional.

${ }^{5}$ En términos del artículo $1^{\circ}$ de la Constitución Federal, las obligaciones de promover, respetar, proteger y garantizar (promover y tomar medidas) los derechos humanos.

${ }^{6}$ En el caso del derecho a la educación superior, los elementos esenciales de accesibilidad, disponibilidad, adaptabilidad y aceptabilidad. ${ }^{7}$ En el orden del derecho mexicano los principios de interpretación conforme a la constitución y los tratados internacionales, y los principios de universalidad, interdependencia, indivisibilidad, progresividad, igualdad y no discriminación y pro persona. A éstos cabe agregar los principios de máximo uso de los recursos disponibles, acceso a mecanismos de exigibilidad, participación y cooperación internacional, derivados de los artículos 1 y 2 de la Declaración Universal de los Derechos Humanos, el art. 2.1 del Pacto Internacional de Derechos Civiles y Políticos, y el art. 2.2 del Pacto Internacional de Derechos Económicos, Sociales y Culturales.
} 
interdependencia y participación, y los principios del interés superior del estudiante, autonomía universitaria y responsabilidad social, propios del ámbito educativo y de la educación superior en particular.

Por el principio de interdependencia se reconoce que los DH entablan entre sí relaciones recíprocas. Éste "señala la medida en que el disfrute de un derecho en particular o un grupo de derechos dependen para su existencia de la realización de otro derecho o de otro grupo de derechos" (Serrano y Vázquez, 2014). En tal sentido, las juntas de gobierno como medio institucional que gravita en torno a la realización del derecho a la ES deben atender al contenido y alcance de éste y al de aquellos derechos interrelacionados con él, dentro de los procesos de designación de la autoridad ejecutiva de la IES a la que pertenezcan.

En el anterior orden de ideas, las juntas de gobierno tienen la responsabilidad de guiarse de tal forma que su conducta institucional sea acorde con el marco constitucional del país y, por ende, satisfaga plenamente, dentro de los procesos de selección de rector(a), los derechos de la comunidad universitaria de participación, petición e información, bajo los principios, en el último caso, de máxima publicidad, transparencia y rendición de cuentas. Asimismo, deben respetar, proteger y garantizar que la libertad de pensamiento, conciencia, expresión, reunión y asociación sean realmente efectivas. Finalmente, quienes forman parte de estos órganos colegiados tienen el deber de conducirse de forma objetiva, imparcial e independiente y con apego a la legalidad, para responder de forma digna al honor que se les ha otorgado, para guarecer la autonomía universitaria y no defraudar la confianza en ellos depositada.

Por otra parte, la participación puede ser abordada como principio transversal de los $\mathrm{DH}$ y como derecho específico. ${ }^{8}$ En su primera faz, es un criterio medular para la construcción del sujeto de derecho, el empoderamiento de la persona y la identificación de necesidades y prioridades de las comunidades (Abramovich y Pautassi, 2006). En la segunda, implica la participación activa, informada y responsable de las personas, en igualdad de condiciones y sin discriminación alguna, en los asuntos que puedan afectarles. Por ende, la toma de medidas para la realización de tal derecho, comprende tanto la adopción de estrategias y medidas que garanticen una amplia participación, como la consulta a las comunidades que podrían verse beneficiadas o afectadas por la toma de decisión correspondiente (Jiménez, 2007).

En el ámbito educativo, diversos órganos y organismos internacionales han subrayado que las comunidades académica y estudiantil son las protagonistas y participantes esenciales de los procesos internos de las IES, por lo que éstas deben elaborar políticas y estrategias que logren su participación activa en todos los aspectos de la vida institucional, y garantizar que sus libertades de pensamiento, conciencia, expresión, reunión y asociación les sean respetadas (UNESCO, 1997, 1998, 2009; OEI, 2008; REDE, 2012).

Además de lo anterior, cabe subrayar que la participación es un componente formativo del derecho a la educación. En efecto, el artículo 13.1 del Pacto Internacional de Derechos Económicos, Sociales y Culturales determina que la educación debe capacitar a todas personas para participar efectivamente en una sociedad libre, favorecer la comprensión y la tolerancia. De ahí que la UNESCO (2009) y el relator especial sobre el Derecho a la Educación de la Organización de las Naciones Unidas (REDE, 2012, 20132, 2014b), insistan en que la educación superior debe contribuir en la formación de ciudadanos dotados de principios éticos y comprometidos con los valores de la democracia, y en que los valores relativos a los DH y los principios democráticos, deben estar en la base

\footnotetext{
${ }^{8}$ Respecto a su carácter de derecho específico, puede verse el art. 25 de la Declaración Universal de los Derechos Humanos, el art. 21 del Pacto Internacional de Derechos Civiles y Políticos y el art. 13.1 del Pacto Internacional de Derechos Económicos, Sociales y Culturales.
} 
de todos los sistemas de educación, pues son parte de una educación de calidad.

Es claro que la formación no se limita a la relación profesor-estudiantes, sino que ella tiene que ver con todo un conjunto de prácticas, modos y formas de interacción entre los miembros de la comunidad universitaria, sus diversos sectores y órganos de administración y gobierno, dentro de los cuales se ubican las mismas juntas de gobierno que con su actuar pueden fortalecer y ampliar la calidad de la participación, coadyuvando con ello en la formación democrática de toda la comunidad universitaria.

Por otra parte, el principio del interés superior del estudiante se enuncia en el artículo 10.c de la Conferencia Mundial sobre la Educación Superior (UNESCO, 1998), aunque ya en 1999 el Comité de Derechos Económicos, Sociales y Culturales (CDESC, 1999), intérprete autorizado del Pacto Internacional del mismo nombre, lo había señalado como criterio para la correcta aplicación de los elementos esenciales ${ }^{9}$ del derecho a la educación. Conforme a tal principio todos los responsables de la adopción de decisiones en el plano institucional deben situar a los estudiantes y sus necesidades en el centro de sus tareas.

La trascendencia del principio del interés superior del estudiante, o del alumno, como también se le conoce, radica en que son precisamente los estudiantes quienes dan sentido, quienes justifican en último término, la existencia de la institucionalidad educativa, al reconocerse socialmente a la educación como uno de los medios más importantes en la formación integral del ser humano. Dicho principio descansa en la centralidad que ocupa en los DH el concepto de dignidad humana (Benjamín, 2010) y el propósito de protección de la persona frente a cualquier abuso del poder, además de reconocer al educando como auténtico titular de derechos y de obligaciones, frente al cual se encuentra el Estado como garante principalísimo del derecho a la ES y las IES como responsables de la aplicación de éste (REDE, 2013).

Lo anterior comporta el deber de las autoridades universitarias, incluidas las juntas de gobierno, de interpretar y aplicar el interés superior del estudiante al momento de asumir decisiones, pues ellas, y en particular la determinación de quién habrá de ser designado rector(a), de una u otra forma tendrá incidencia en la garantía a los estudiantes del disfrute efectivo del derecho a la ES y de los derechos interrelacionados con él.

Finalmente, la autonomía universitaria y la responsabilidad social de las IES son principios que vale la pena tratar de forma conjunta, ya que el primero implica al segundo y ambos van necesariamente unidos (OEI, 2008). La autonomía consiste en el grado de autogobierno necesario para que las IES adopten decisiones eficaces, en relación con sus actividades académicas, su normativa, sus trabajos administrativos, de gestión y actividades conexas (UNESCO, 1997; CDESC, 1999). Ella es una condición necesaria para el adecuado desempeño de las funciones encomendadas al personal académico y a las IES, y para que éstas mantengan una clara independencia política, ética y científica (UNESCO, 1996).

No obstante, la autonomía debe ejercerse bajo el principio de responsabilidad social y, en ese sentido, las IES deben lograr un equilibrio apropiado entre la autonomía institucional y sus sistemas de rendición de cuentas, mismos que obligan a contar con una gestión transparente, a informar sobre su misión educativa, a velar por la calidad de sus funciones sustantivas, a proteger y garantizar la integridad de sus funciones ante toda injerencia incompatible con su misión académica, a respetar y proteger los DH y las libertades fundamentales, y a vigilar que sus funciones se realicen conforme a las normas éticas aplicables (UNESCO, 1997, 1998).

\footnotetext{
${ }^{9}$ Respecto a estos elementos, véase la nota número 6.
} 
Como se observa, generar un acercamiento desde un enfoque de $\mathrm{DH}$ a la atribución de las juntas de gobierno de seleccionar al rector(a) de las IES autónomas, significa centrar la atención en el derecho a la ES como aquel que debe orientar el ejercicio de tal potestad. Y, tal como se ha visto, éste, en su carácter de derecho humano, exige el respeto, la protección y garantía de otros tantos derechos, así como la transparencia y la rendición de cuentas. Así pues, la selección de la autoridad ejecutiva de las IES autónomas no cae de ninguna forma bajo el manto de la discrecionalidad, en manos de un pequeño grupo de personas, sino en el campo abierto del cumplimiento de los derechos y de la obligación de adoptar decisiones en pro del derecho a la ES, y de ésta superior como un bien público.

\section{El proceso de designación rectoral 2017 de la Universidad Veracruzana}

En el primer apartado se expuso que el modelo de selección de rector(a) por juntas de gobierno es criticado por observarse en él rasgos autoritarios y de secrecía, porque a partir de la praxis se percibe la subordinación de éstas a grupos de interés internos y/o externos (Ordorika et al., 2011; Ordorika, 2015; Vargas y Olivares, 2007) y porque, entre otras cosas, se piensa que su trabajo se ha traducido en la disminución o pérdida del dinamismo en la participación de la comunidad universitaria (Abello, 2015; López, 2013; Ramírez, 2013).

La Junta de Gobierno de la UV llevó a cabo su más reciente proceso de designación en 2017, del 30 de junio al 31 de agosto. Teniendo presentes los déficits que acusaba a la luz de los estudios existentes en torno a los órganos colegiados que, como ella, tienen la tarea de designar al rector(a) de IES autónomas, realizó una serie de innovaciones con el propósito de responder a aquello que como mínimo deben garantizar las juntas de gobierno en el marco del derecho a la ES.

En tal orden de ideas, la Junta editó un libro blanco del proceso, promovió una entrevista estandarizada de cada una de las personas que aspiraron al cargo de rector(a), organizó una presentación pública de las propuestas de plan de trabajo de los candidatos(as) a la rectoría, amplió la auscultación a la comunidad universitaria a través de medios electrónicos y creó una plataforma de información del proceso de designación rectoral. Estos mecanismos y acciones tuvieron una incidencia positiva sobre la realización de derechos específicos de los integrantes de la comunidad universitaria, como se verá a continuación, pero también mostraron áreas de oportunidad y retos que deberían ser tenidos en cuenta en los futuros ejercicios de designación rectoral.

La Ley de Autonomía de la UV obliga a su Junta (art. 8, fracción I, inciso d) a publicar la convocatoria de consulta a la comunidad universitaria, dos meses antes de la conclusión del periodo del rector(a) en funciones, lo cual entraña una seria limitación de tiempo para llevar a cabo las diferentes etapas del proceso, sobre todo tomando en cuenta que una parte del lapso del que dispone la Junta, empalma con el periodo vacacional de verano de la Universidad. Esta anotación marginal ayuda a comprender por qué la Junta de Gobierno en funciones decidió elaborar el libro blanco, denominado $A B C$ del proceso de designación rectoral de la Universidad Veracruzana (Junta de Gobierno de la Universidad Veracruzana, 2017), pues el mismo constituía, sin duda, una vía idónea para la satisfacción del derecho a la información de los universitarios de esa casa de estudios.

El derecho a la información consagrado en el artículo 6 de la Constitución federal y en su homólogo de la Constitución veracruzana (art. 6, sexto párrafo), comprende el derecho a distribuir información, a acceder a ella y a recibirla. Asimismo, es un derecho que, en el ámbito de la educación superior, debe garantizar que todos los universitarios cuenten con la información que incide en el ejercicio de sus derechos. Por ende, el deber de las juntas de gobierno de informar sobre las funciones que realizan no se circunscribe al periodo durante el cual realizan sus 
procesos de designación, este deber es permanente y la información sobre las tareas que llevan a cabo pueden proveerla en cualquier momento e, incluso, de forma continua. No obstante, y en virtud de que nunca antes se había hecho un ejercicio de tal naturaleza en la veracruzana, la Junta de Gobierno editó en 2017 el $A B C$ del proceso de designación rectoral y lo presentó a los universitarios interesados en el tema en las cinco regiones que integran la Universidad, del 13 al 23 de marzo.

$\mathrm{El}$ texto que se comenta, dio a conocer los derechos de la comunidad universitaria dentro del proceso de designación y las reglas y principios bajo los cuales debía desarrollarse; los criterios que regirían en la auscultación a la comunidad, así como las innovaciones que se aplicarían y las obligaciones a cargo de los diversos participantes, incluida la propia Junta de Gobierno y sus integrantes. El esfuerzo por satisfacer el derecho a la información de la comunidad universitaria también incluyó la generación de un resumen del $A B C$ del proceso de designación rectoral, una infografía sobre las fechas relevantes del proceso, la emisión de boletines y comunicados en torno al mismo, y la publicación de la convocatoria de ley, junto con los criterios que habrían de aplicarse en la revisión y valoración de expedientes de los aspirantes a la rectoría; en la auscultación a la comunidad, y en la entrevista a realizar por el pleno de la Junta a quienes fuesen elegidos como candidatos a rector(a). Elementos todos a los que la comunidad pudo acceder a través de la plataforma de información oficial del proceso, sobre la cual se profundizará más adelante.

Con antelación se dijo que el derecho a la información debe ser satisfecho en observancia de los principios de máxima publicidad, transparencia y rendición de cuentas. Es cierto que, como anteriormente se expuso, la designación de la máxima autoridad ejecutiva de las IES autónomas es una atribución de las juntas de gobierno, pero como se ha argumentado, ésta debe ejercerse en el marco del derecho a la ES. Es en la lógica de un auténtico Estado constitucional y democrático de derecho que los principios citados cobran mayor significación, pues las comunidades universitarias tienen el derecho a que las juntas ejerzan su atribución en cumplimiento de las reglas y principios que les impone el orden constitucional y legal del país. Así, la publicidad y la transparencia de su actuar ayudan en la rendición de cuentas, respecto al ejercicio de una atribución delicada y trascendente en la vida institucional de las IES autónomas. Dar publicidad y transparentar los elementos de juicio con los que cuentan las propias juntas de gobierno, provee a las comunidades universitarias de elementos para valorar su desempeño. En este sentido, en el proceso de designación que se comenta, la plataforma de información ${ }^{10}$ pretendió ser una caja de cristal, a través de la cual cualquier universitario(a) interesado(a) en el desarrollo del proceso pudiese acceder a todo elemento o insumo que en torno a él se generase.

Así, la plataforma electrónica de la Junta de Gobierno de la UV, incorporó todos los elementos de información que fueron indicados en párrafos anteriores y puso a disposición del público en general los currícula, las propuestas de plan de trabajo y las respuestas proveídas por los aspirantes a la rectoría a una encuesta, teniendo la oportunidad, cualquier visitante de la página web, de comparar los resultados de esta última entre los diferentes aspirantes. Además, en la plataforma se integraron las grabaciones de la presentación del $A B C$ del proceso de designación rectoral, de la entrevista estandarizada a los aspirantes y de la presentación de las propuestas de planes de trabajo, con el objetivo de que quienes no tuvieron oportunidad de asistir a cada uno de los eventos indicados pudiesen acceder a la información proporcionada en ellos.

En un hecho que bien puede ser calificado de inédito, en el contexto de las IES autónomas, la página

${ }^{10} \mathrm{El}$ contenido alojado en la plataforma de información, durante el proceso de designación rectoral 2017, puede consultarse en: https://www.uv.mx/juntagob/procesorectoral2017/. 
web de la Junta de Gobierno publicó los resultados obtenidos en la auscultación a la comunidad universitaria, la evaluación curricular y de las propuestas de plan de trabajo de cada uno de los aspirantes a la rectoría. Asimismo, publicó las cédulas de evaluación de cada uno de los integrantes de la Junta, respecto a la entrevista que realizó el pleno a cada una de las personas que alcanzaron el carácter de candidatos a la rectoría. ${ }^{11}$ Aquí interesa señalar que las entrevistas a los candidatos(as) se ajustaron en todo momento a las condiciones de tiempo, orden, lugar, mecánica y temáticas, que previa y oportunamente les fueron dadas a conocer a éstos y que respondieron en su programación al principio de igualdad. Como es de destacar, el empeño por dar publicidad y por transparentar el proceso fue significativo e implicó un avance cualitativo respecto a ejercicios anteriores, contando la comunidad universitaria con mayores elementos de juicio para valorar el desempeño de su Junta y para comprender, en una mayor medida, el régimen legal e institucional que rige la designación de su autoridad ejecutiva.

La plataforma de información contó, como se indicó, con una herramienta comparativa de la información proveída por las y los aspirantes al cargo. Ésta se diseñó como un elemento adicional que permitiera que las percepciones y opiniones de cada aspirante sobre diversos temas del quehacer y la problemática universitaria, fuesen puestos todos a la vez, según la temática plateada (nivel académico, calidad de los productos de investigación, etcétera), de tal forma que la percepción de los aspirantes sobre el tópico (buena, regular, mala), las razones del por qué percibirlo así y su propuesta de mejora, fuesen visualmente comparables por los visitantes de la plataforma. Este mosaico comparativo propició que los visitantes contaran, además de con la información individual, con la percepción global y la argumentación que se proveía en torno a un tema. En tal orden de ideas, el apartado contribuyó a que los miembros de la comunidad universitaria evaluaran las percepciones y las propuestas de los y las aspirantes, para la mejora en las funciones sustantivas de la UV.

En adición a lo anterior, cabe decir que con la información obtenida de las opiniones de los aspirantes y las respuestas al cuestionario respondido por los miembros de la comunidad universitaria, se creó un índice denominado Índice de coincidencia. Para esta construcción se consideró la categoría con el mayor porcentaje de las percepciones de la comunidad universitaria participante en cada ítem y éste se contrastó con la categoría asignada por cada aspirante, las coincidencias se sumaron y se reescalaron a un valor entre 1 y 10 , obteniéndose así el indicador de acuerdo entre percepción de aspirante y percepción de la comunidad universitaria. De tal forma que los integrantes de la comunidad tuvieron la oportunidad de visualizar qué tan cercanas o qué tan lejanas eran sus propias percepciones de las de cada uno de los aspirantes al cargo de rector(a).

El uso de la plataforma también permitió recabar, integrar y sistematizar la opinión de la comunidad universitaria y detectar necesidades, prioridades y problemáticas percibidas, mismas que fueron dadas a conocer al público en general a través del mismo medio y entregadas a la administración universitaria entrante, lo que implicó una acción de rendición de cuentas por parte de la Junta de Gobierno y proveyó de insumos a la comunidad universitaria para dar seguimiento a las labores realizadas por su nueva administración. En este sentido, vale la pena tener presente el derecho fundamental a una buena administración, reconocido en la Carta Iberoamericana de los Derechos y Deberes del Ciudadano en relación con la administración pública (Centro Latinoamericano de Administración para el Desarrollo, 2013).

\footnotetext{
${ }^{11}$ Al proceso de designación rectoral 2017 de la UV se presentaron un total de siete aspirantes, cuatro hombres y tres mujeres. En términos de los artículos 6, 20, 21 y 24 del Reglamento de la Junta de Gobierno, el órgano colegiado seleccionó a las tres personas, dos mujeres y un hombre, que consideró idóneas para convertirse en candidatos(as) y para competir, por lo tanto, por el cargo de rector(a).
} 
Con antelación se señaló que los integrantes de las juntas de gobierno deben conducirse con legalidad, objetividad, imparcialidad e independencia. El hecho de que las juntas publiquen todos y cada uno de los elementos que se relacionan con el proceso de designación rectoral o que han sido generados durante el mismo, es un incentivo para que sus miembros ciñan su conducta a tales principios, pues la motivación y la legitimidad de ésta, serán leídas por la comunidad universitaria a partir de los elementos de juicio existentes, pues a ellos tienen acceso todos los integrantes de ésta y la sociedad en general. En tal tesitura se piensa que aún se dispone de otras estrategias posibles para garantizar los principios a los que deben respeto los miembros de las juntas de gobierno, pero sobre ellas se abundará en el siguiente apartado.

En relación con lo desarrollado en el párrafo anterior, sólo baste con realizar una anotación más, a nuestro juicio, entre mayor publicidad y transparencia exista en los procesos de designación del rector(a) de las IES autónomas, menores son los riesgos de vulneración de la autonomía universitaria, pues la intromisión indebida podría ser detectada a partir de la disonancia entre los elementos de juicio objetivos y la decisión adoptada por las juntas de gobierno (Abello, 2015). En este sentido, es de señalar el movimiento surgido en la Universidad Nacional Autónoma de México (UNAM) a propósito de la designación rectoral que habría de llevar a cabo su Junta de Gobierno en 2015, pues justo en él, los académicos involucrados, muchos de ellos destacados especialistas en educación superior, exigieron transparencia y rendición de cuentas por parte de su Junta de Gobierno, en pro del aseguramiento de la autonomía de la UNAM (Aguilar, 2015).

Por otra parte, los medios de participación a disposición de la comunidad universitaria de la UV se ampliaron de forma significativa, pues se transitó de un sólo mecanismo de participación, con serias limitaciones de tiempo, esto es, la auscultación directa a la comunidad, a la posibilidad de participar a través de una encuesta en línea, del envío de comentarios a la Junta y del planteamiento de preguntas que podrían ser formuladas aleatoriamente a los candidatos a la rectoría, en la presentación de sus propuestas de plan de trabajo. Este conjunto de acciones favoreció en gran medida el ejercicio de las libertades fundamentales de pensamiento y expresión, que, como se vio al inicio de este documento, son derechos interdependientes del derecho a la ES.

Por último, un par de elementos que pueden ser destacados dentro del proceso de designación rectoral que se expone, son el comunicado a la comunidad universitaria sobre la decisión asumida por la Junta de Gobierno respecto a la designación del rector y el proceder de ésta para la garantía del derecho de petición de los miembros de la misma comunidad. En relación al primer punto cabe decir que, derivado de las reformas operadas al RJG en mayo de 2014, ${ }^{12}$ el comunicado de la Junta de Gobierno fue mucho más extenso que cualquier otro de los anteriores procesos, pues no se limitó a señalar el resultado y las normas legales en que sustentó su decisión, sino que expuso de forma pormenorizada las acciones emprendidas para la asunción de su determinación, dio a conocer el resultado de la votación interna del órgano colegiado y expuso las razones que sustentaron su decisión final; en tal sentido, la comunidad universitaria de la UV tuvo acceso a un texto que explica en detalle la forma en la que se despliga el proceso de designación del rector en su institución y conoció el porqué de la decisión mayoritaria del órgano colegiado. Por cuanto hace al segundo punto, sólo baste con señalar que la Junta de Gobierno dio respuesta a todas y cada una de las observaciones,

\footnotetext{
${ }_{12}$ Puede consultarse al respecto el artículo 32 del Reglamento de la Junta de Gobierno de la Universidad Veracruzana y el histórico de sus reformas y modificaciones que aparece al final del mismo.
} 
inconformidades y peticiones planteadas por escrito al órgano colegiado por integrantes de la comunidad universitaria, algunas de las cuales fueron publicadas en medios informativos locales, electrónicos e impresos, pero a las cuales la Junta de Gobierno dio respuesta de forma personalizada; todos los peticionarios decidieron, hasta el momento en el que se trazan estas líneas, mantener en el ámbito privado las respuestas que por escrito les hizo llegar la Junta de Gobierno.

\section{Hallazgos y áreas de oportunidad}

A pesar de la diversidad de mecanismos instaurados durante el proceso, la participación de la comunidad fue poca o, por lo menos, menor a la expectativa que se tenía en torno a ella. En la auscultación directa, es decir, en las entrevistas cara a cara que realizaban miembros de la comunidad con integrantes de la Junta, participaron 1344 personas, quedando lugares para entrevistas sin solicitar. La plataforma de información fue visitada 58979 veces, en una comunidad universitaria de 97266 miembros, al momento del proceso de designación (Dirección de Planeación Institucional, 2017); 423 universitarios dieron respuesta a la encuesta en línea promovida por la Junta de Gobierno, 90 enviaron comentarios a la misma y 124 formularon preguntas a los candidatos a la rectoría.

Para conocer las razones por las que se dio una participación menor a la esperada, éstas tendrían que buscarse a través de una investigación específica. No obstante, la literatura especializada (López, 2013) ha identificado, mediante la técnica de entrevista, una serie de situaciones que podría tener incidencia respecto al tema. Se dice, por ejemplo, que algunos sectores universitarios, en especial el del personal académico, piensan que el proceso de designación rectoral por juntas de gobierno:

- Se resuelve, en los hechos, en instancias externas a la Universidad.
- Permite la apropiación de la rectoría por parte de grupos profesionales o de interés.

- Toma en cuenta factores no académicos para la designación del rector(a).

- Supone que la comunidad universitaria sabe de antemano quién será el ganador, por lo que se organiza la "cargada" a favor de éste.

- No es una auténtica competencia, sino una mera formalidad que valida decisiones asumidas en otros ámbitos, esto es, existen grupos de poder y de presión con la capacidad de imponer a un candidato(a) manipulando la normativa institucional a su conveniencia (López, 2013).

Opiniones e ideas como las anteriores, muestran escepticismo respecto a los procesos de designación rectoral llevados a cabo por las juntas de gobierno y bien explican, pero no justifican, la ausencia o la poca participación de la comunidad universitaria, pues como bien se sabe, la participación es, a la vez, un derecho y una obligación y, asimismo, es una condición necesaria para que las juntas de gobierno cuenten con insumos apropiados para la toma de decisiones.

Un par de elementos más que vale la pena destacar como hallazgos, son la desconfianza en que realmente funcionasen los mecanismos prometidos por la Junta durante el proceso y el que la información proveída en la auscultación, ya fuese directa o a través de la plataforma de información, fuese a mantenerse reservada. Afirmaciones que se desprenden tanto de comentarios aislados de integrantes de la comunidad universitaria de la UV, como del uso que algunas personas hicieron de la plataforma, al utilizar los dispositivos a su alcance, en las secciones de envío de comentarios y de preguntas, para remitir mensajes en blanco o vacíos.

Un último hallazgo, que engarza con las áreas de oportunidad que se detectan, es que la información proveída por las juntas de gobierno, ligada a los principios constitucionales de máxima publicidad y 
de transparencia, podría ampliarse aún más. A este respecto, de nueva cuenta es relevante el debate desarrollado en 2015 en la UNAM (Aguilar, 2015), pues a partir de él también se solicitó a la Junta respectiva (Gómez, 2015a) que difundiera mediante transmisión televisiva, radiofónica o electrónica las comparecencias de los aspirantes con la Junta de Gobierno y las deliberaciones que llevasen a cabo los integrantes de la Junta para la elección del rector(a).

Como era de esperarse la opinión universitaria se dividió, incluso entre los expertos en la temática (Gómez, 2015a), arguyendo quienes no estaban de acuerdo con la implementación de la propuesta, que con ésta se expondría al proceso a presiones externas e implicaría un riesgo para que éste no fuese libre, independiente y aceptado por todos. A este respecto, lo primero que debe destacarse es que el que las deliberaciones de las juntas de gobierno o las entrevistas a aspirantes a la rectoría sean reservadas, no excluye la posibilidad de que los integrantes de estos órganos colegiados sean sometidos a presiones de grupos de poder o de interés o que su participación carezca de independencia e imparcialidad. Tampoco asegura que la decisión asumida por las juntas sea aceptada o bien vista por todos(as).

En segundo lugar, es de llamar la atención que por disposición constitucional (art. 28, 41, 94 y 99) las sesiones de las salas y del pleno de la SCJN, de la sala superior y regionales del Tribunal Electoral, de los órganos de gobierno de la Comisión Federal de Competencia Económica y del Instituto Federal de Telecomunicaciones, así como de todos los órganos colegiados de dirección del Instituto Nacional Electoral son públicas y, sólo por excepción, que debe estar reconocida en la ley, pueden ser privadas. Estas normas son relativamente recientes pues obedecen a reformas que se dan entre 2013 y 2014, y tienen como objetivo transparentar el ejercicio público y hacer plenamente efectivo el derecho fundamental de acceso a la información. ¿Por qué las deliberaciones que llevan a cabo los integrantes de las juntas de gobierno en los procesos de designación rectoral o las entrevistas que realizan a candidatos a la rectoría deberían permanecer privadas? En realidad no se vislumbra un argumento sólido que pueda sustentar el carácter secreto de las entrevistas hechas por los plenos de las juntas a los candidatos a la rectoría y de las deliberaciones entre integrantes de las juntas para la designación del rector(a). Si órganos de mucho mayor jerarquía, y que tratan asuntos que es posible suponer involucran intereses de sectores con un mayor peso económico y/o político, han avanzado hacia la deliberación pública, ¿por qué las juntas de gobierno no?

En realidad si, como se ha argumentado en este escrito, las juntas de gobierno deben ejercer su atribución de designar a la persona que habrá de ocupar la rectoría en el marco del derecho a la ES, entonces sus deliberaciones deben ceñirse a la máxima satisfacción de éste y atender a la evidencia con la que cuenten para asumir su decisión. Sus discusiones deben orientarse a identificar al mejor candidato(a) a la rectoría (conforme a su normativa interna), no tienen un objetivo político, ni persiguen generar un apoyo democrático (SCJN, 2014). El objetivo de las sesiones públicas es entonces que los universitarios, y la sociedad en general, observen un debate entre los integrantes del órgano colegiado, en el que cada uno intentará persuadir, mediante argumentos, a sus compañeros de su postura, lo cual permitiría al público en general conocer la opinión de cada uno de los miembros de la Junta y contribuiría - contrario a lo que se sostiene - con la independencia de sus integrantes, favorecería su esmero en la atención de la delicada tarea que han asumido y permitiría a la comunidad universitaria evaluar su desempeño, lo que en conjunto, y en condiciones ideales, acrecentaría la confianza en estos órganos colegiados por parte de la comunidad universitaria y de la sociedad en general (Diario Oficial de la Federación, 2016), algo que, como se ha visto a lo largo de este documento, no abunda. Esto parece haberlo percibido con toda claridad la 
Junta de Gobierno de la Universidad Autónoma de Baja California (UABC), pues es la primera en su tipo que ha demostrado la voluntad de ser plenamente transparente en sus procesos de designación, al transmitir en vivo, a través de su canal universitario, todas sus sesiones de trabajo dentro del proceso de designación rectoral (UABC, 2014).

En íntima relación con lo anterior se encuentra el derecho a disentir, pues aun cuando políticamente se piense que las decisiones unánimes respecto a la designación de rector(a) fortalece la posición institucional de las IES autónomas, bien es cierto que esto no es coincidente con el marco constitucional nacional de los DH ni con la forma de abordar los deberes de respeto, protección y garantía de éstos. Como bien se dice en la jerga jurídica, cuando la mayoría es el criterio de decisión, la razón o los argumentos salen sobrando. Por ello, Ferrajoli, destacado y controvertido filósofo del derecho, ha sostenido que los derechos fundamentales deben sustraerse de las decisiones de la mayoría, pues son derechos que se afirman siempre como leyes en alternativa a la ley del más fuerte (Ferrajoli, 1999). Ajustado lo anterior a los fines de este texto, puede expresarse en los siguientes términos: en la normalidad democrática no tienen por qué estar todos de acuerdo ni tienen por qué fingir tal acuerdo, porque el disenso expresa la buena salud de cualquier sociedad auténticamente democrática. El disenso, en el caso que nos ocupa, tampoco expresa un desacuerdo sobre los fines esenciales que deben ser protegidos, los cuales, dicho sea de paso, vienen dados por nuestro orden constitucional y legal, sino sólo sobre quién es la persona idónea, a juicio de los integrantes de las juntas de gobierno, para su respeto, promoción y defensa. Un juicio que, claro está, debería poder desempeñarse argumentativamente.

Finalmente, partimos de la firme convicción de que un "argumento" que no es posible mantener ante el público en general o que no se desea sostener ante el mismo, no constituye un auténtico argumento.

Como se indicó anteriormente, la Junta de
Gobierno de la UV publicó en su plataforma, junto con la convocatoria de ley, los criterios aplicables para la valoración de expedientes, la auscultación directa y la entrevista a realizar por el pleno de la Junta a quienes fuesen elegidos como candidatos a rector(a). A la luz de la experiencia adquirida se observa que no sólo deberían ser publicados los criterios que habrá de utilizar el órgano colegiado, sino también los instrumentos concretos que habrán de aplicarse, de tal forma que se provea de plena seguridad jurídica a quienes pretenden participar en el proceso.

Por cuanto hace a la plataforma de información y las herramientas que en ella podrían incorporarse, observamos que en ejercicios futuros sería deseable que la Junta de Gobierno de la UV realice una amplia consulta que propicie una revisión de los apartados de los cuestionarios, derivadas de una participación responsable y comprometida de toda la comunidad y, en particular, de los miembros de los diversos cuerpos colegiados de la Universidad. Asimismo, consideramos que habría que comunicar con una mayor amplitud y eficacia los elementos de análisis de los datos obtenidos de la participación de la comunidad universitaria y que sería deseable que los instrumentos de evaluación aplicados en las etapas finales del proceso, sean valorados desde la perspectiva de la confiabilidad y validez, características que a todo instrumento de evaluación debe acompañar. También pensamos que los elementos técnicos de capacidad de proceso y velocidad de acceso y de respuesta deben ser revisados y actualizados de forma constante.

Consideramos, asimismo, que la participación de la comunidad universitaria como lo propició la Junta, en las modalidades de consulta directa e indirecta, pueden dar pauta a que en un futuro se consolide un sistema de información y de seguimiento, a compromisos y acciones realizados por la persona que resulte elegida como rector(a) de la Universidad.

Finalmente, entendemos que en los casos de IES autónomas que contemplan la posibilidad de 
reelección de su rector(a), una tarea que deberían asumir las juntas de gobierno es la de recabar la percepción de la propia comunidad universitaria respecto al desempeño de la persona que ocupó la rectoría, así como de la administración que estuvo a su cargo. Este estudio debería complementarse con una evaluación externa, independiente e imparcial, sobre el desempeño de la persona que esté legalmente en posibilidad de reelegirse y de la administración saliente a su cargo, en relación con los principales componentes del derecho a la ES, de los principios que le rigen y, en general, del respeto de los derechos y de las libertades fundamentales de la comunidad universitaria. De tal forma que los integrantes de estos órganos colegiados cuenten con elementos objetivos de juicio que les permita, en la medida de lo humanamente posible, adoptar la mejor decisión respecto a la comunidad universitaria en general y en especial de la comunidad estudiantil que, como se ha visto, constituye la razón última de la existencia de la educación superior y de los diversos sectores que conforman a la comunidad universitaria.

\section{Conclusión}

Tal como acertadamente lo ha dicho Ordorika (2015), no cabe duda alguna de la necesidad de que las IES públicas y autónomas lleven a cabo un esfuerzo de discusión y elaboración de alternativas para transformar los mecanismos de selección de sus rectores (no sólo del método de selección por juntas de gobierno), que permitan superar los rezagos existentes y desviaciones, y que generen condiciones apropiadas para el establecimiento de mecanismos transparentes.
Es un hecho, teorizado e ilustrado por la doctrina (Ramírez, 2013; Ordorika et al., 2011; Ordorika, 2015; Vidal y Vieira, 2014), que las IES son producto de las contradictorias condiciones materiales de las formaciones sociales a las que pertenecen, las cuales suelen reproducir institucionalmente, en conciencia o no. Quizá ello explique, en buena medida, el por qué todos los métodos de selección de rector(a) estén bajo sospecha y se les observe como un formalismo, alejado del espíritu de las legislaciones universitarias (López, 2013).

Sería ingenuo pensar que las IES mexicanas se mantienen impolutas en el contexto de la debilidad institucional, democrática y de la cultura de la legalidad que aquejan al Estado mexicano. De ahí que estimemos que las juntas de gobierno de las universidades públicas autónomas tienen ante sí el reto, en sí mismo altamente estimulante para la inteligencia sensible, humanista, creativa y comprometida que encarna en miles de universitarios del país, de generar procesos de designación rectoral con los que, si bien no todos sean felices con el resultado, si generen seguridad sobre sus procesos y la legitimidad institucional que se requiere para hacer frente a los complicados tiempos que enfrenta la educación superior pública en México.

Finalmente, no podría cerrarse este apartado conclusivo sin apuntar a que cualquier innovación que emprendiesen las juntas de gobierno en aras del ejercicio de los derechos de las comunidades universitarias, de la transparencia y la rendición de cuentas, sería poco efectiva si no es acompañada de una participación comprometida, responsable y madura de todos los sectores universitarios. 


\section{Referencias}

Abello, Juan (2015), "Sistemas de gobierno en las universidades de Latinoamérica: la importancia de la composición de los máximos cuerpos colegiados", Revista chilena de economía y sociedad, vol. 9, núm. 2, pp. 30-47.

Abramovich, Víctor y Laura Pautassi (2006), "Dilemas actuales en la resolución de la pobreza. El aporte del enfoque de derechos", Ponencia presentada en el Seminario taller: los derechos humanos y las políticas públicas para enfrentar la pobreza y la desigualdad, Buenos Aires, UNESCO, disponible en: <http:// docplayer.es/12280992-Dilemas-actuales-en-laresolucion-de-la-pobreza-el-aporte-del-enfoque-dederechos.html> [Consulta: octubre de 2017].

Acosta, Adrián (2014), "Gobierno universitario y comportamiento institucional: la experiencia mexicana, 1990-2012", Bordón. Revista de pedagogía, vol. 66, núm. 1, pp. 31-44.

Aguilar, Andro (2015), "UNAM: hora crítica", Reforma, México, 25 de octubre, disponible en: <http://www. reforma.com/aplicacioneslibre/articulo/default.aspx? $\mathrm{id}=675855 \& \mathrm{md} 5=9 \mathrm{c} 5 \mathrm{f} 2030 \mathrm{f} 21 \mathrm{bca} 5$ fada $0 \mathrm{ad} 6008 \mathrm{f} 71$ $6 c d \& t a=0 d f d b a c 11765226904$ c16cb9ad1b2efe\&lcmd $5=4 \mathrm{e} 5 \mathrm{f} 4 \mathrm{c} 56 \mathrm{~d} 4 \mathrm{ed} 2 \mathrm{cbb7e90be901df7f26c> \text {[Consulta: }}$ noviembre de 2017].

Benjamín, Ramón (2010), "Educación y estado de derecho", Manos y cerebros, Panamá, Asociación de Educadores Veragüenses, disponible en: <https:// manoscerebros.wordpress.com/2010/12/03/educaciony-estado-de-derecho/> [Consulta: octubre de 2017].

Casanova, Hugo y Roberto Rodríguez (2014), "Universidad, política y gobierno: vertientes de interpretación y perspectivas de análisis", Bordón. Revista de pedagogía, vol. 66, núm. 1, pp. 151-164.

Comité de Derechos Económicos, Sociales y Culturales (CDESG) (1999), Observación general número 13. El derecho a la educación (artículo 13 del Pacto, E/C.12/1999/10), Ginebra, Consejo de Derechos Humanos de la Organización de las Naciones Unidas.
Centro Latinoamericano de Administración para el Desarrollo (2013), Carta iberoamericana de los derechos y deberes del ciudadano en relación con la administración pública, Panamá, Centro Latinoamericano de Administración para el Desarrollo.

Constitución Política del Estado de Veracruz de Ignacio de la Llave (2017), Gaceta Oficial del Estado de Veracruz, Veracruz, 23 de noviembre.

Contreras, Rebeca, Jaqueline Jongitud, Martha Daniels, María Montalvo, Socorro Moncayo y Josefa Montalvo (2013), "Derecho a la enseñanza superior", en Josefa Montalvo (coord.), Estudios multidisciplinares de derecho y globalización, México, Centro de Estudios sobre Derecho, Globalización y Seguridad de la Universidad Veracruzana, pp. 203-236.

Diario Oficial de la Federación (2016), Acuerdo general del pleno del Consejo de la fudicatura Federal que reforma y adiciona el similar 16/2009, que regula las sesiones de los Tribunales Colegiados de Circuito y establece los lineamientos para su videograbación y difusión, México, 28 de enero, disponible en: <http://www.dof.gob.mx/nota_detalle.php?c odigo $=5423814 \&$ fecha $=28 / 01 / 2016>$ [Consulta: noviembre de 2017].

Dirección de Planeación Institucional (2017), uv en números, Veracruz, Universidad Veracruzana, disponible en: <https://www.uv.mx/informacion-estadistica/ files/2014/01/UV_en_numero_agosto_2017.pdf> [Consulta: septiembre de 2017].

Ferrajoli, Luigi (1999), Derechos y garantías. La ley del más débil, Madrid, Trotta.

Gómez, Natalia (2015a), "Debate por método para elegir rector en la UNAM", en El Universal, México, 6 de agosto, disponible en: <http://www.eluniversal. com.mx/articulo/nacion/sociedad/2015/08/6/ debaten-por-metodo-para-elegir-al-rector-de-launam> [Consulta: noviembre de 2017].

Gómez, Natalia (2015b), "Difieren universidades en métodos para elegir rector", El Universal, México, 10 de agosto, disponible en: <http://www.eluniversal. 
com.mx/articulo/nacion/sociedad/2015/08/10/ difieren-universidades-en-metodos-para-elegirrector> [consulta: noviembre de 2017].

Jiménez, William (2007), "El enfoque de los derechos humanos y las políticas públicas", Civilizar, vol. 7, núm. 12, pp. 31-46.

Jongitud, Jaqueline (2017), "El derecho humano a la educación superior en México", Revista de la Educación Superior, vol. 46, núm. 182, México, Asociación Nacional de Universidades e Instituciones de Educación Superior (ANUIES), pp. 45-56.

Jongitud, Jaqueline (2014), "Por una ley general de educación superior para México", Letras jurídicas, vol. 30, núm, 15, pp. 59-75.

Junta de Gobierno de la Universidad Veracruzana (2017), abc del proceso de designación rectoral de la Universidad Veracruzana, Xalapa, Universidad Veracruzana, disponible en: <https://www.uv.mx/juntagob/procesorectoral2017/> [Consulta: noviembre de 2017].

Ley de Autonomía de la Universidad Veracruzana (2017), Xalapa, Universidad Veracruzana.

López, Romualdo (2014), "La interacción gobiernouniversidades: una relación compleja”, Bordón. Revista de pedagogía, vol. 66, núm. 1, pp. 75-88.

López, Romualdo (2013), "Percepciones de académicos, directivos y funcionarios sobre la legitimidad y el liderazgo de los rectores de las universidades públicas mexicanas", Revista Mexicana de Investigación Educativa, vol. 18, núm. 58, pp. 811-832.

López, Romualdo (2010), "Normatividad, formas de gobierno y gobernabilidad en las universidades públicas mexicanas: ¿será posible su transformación?”, en Daniel Cazés, Eduardo Ibarra y Luis Porter (coords.), Las universidades públicas mexicanas en el año 2030: examinando presentes, imaginando futuros, México, CIIIH-UNAM/UAM Guajimalpa.

López, Romualdo, Óscar González, Javier Mendoza y Judith Pérez (2011a), "Las formas de elección de los rectores. Otro camino para acercarse al conocimiento de las universidades públicas autónomas", Perfiles Educativos, vol. XXXIII, núm. 131, pp. 8-27.

López, Romualdo, Óscar González, Javier Mendoza y Judith Pérez (2011b). "El rol de los rectores en la gobernabilidad de las universidades públicas mexicanas", Revista Mexicana de Investigación Educativa, vol. 16, núm. 51, pp. 1021-1054.

Organización de Estados Iberoamericanos (OEI) (2008), Declaración de la Conferencia Regional de Educación Superior en América Latina y el Caribe, Cartagena de IndiasColombia, OEI.

Ordorika, Imanol (2015), "Elección de rector: panorama internacional", Revista de Educación Superior, vol. XLIV (3), núm. 175, pp. 7-18.

Ordorika, Imanol (2014), "Governance and change in higher education: the debate between classical political sociología, new institutionalism and critical theories", Bordón. Revista de pedagogía, vol. 66, núm. 1, pp. 107-122.

Ordorika, Imanol,Jorge Martínez y Rosa María Ramírez (2011), "La transformación de las formas de gobierno en el sistema universitario público mexicano: una asignatura pendiente", en Revista de Educación Superior, vol. XL (4), núm. 160, pp. 29-49.

Ramírez, Rosa (2013), "El gobierno universitario ¿un poder de Estado en educación?", Revista de psicología de la Universidad del Estado de México, vol. 2, núm. 3, pp. 33-54.

Relator Especial sobre el Derecho a la Educación (REDE) (2014a), Informe del relator especial sobre el derecho a la educación, A/71/58, Ginebra, Consejo de Derechos Humanos de la Organización de las Naciones Unidas. REDE (2014b), Informe del relator especial sobre el derecho a la educación, Kishore Singh. Evaluación del logro educativo de los estudiantes y de la aplicación del derecho a la educación, A/HRC/26/27, Ginebra, Consejo de Derechos Humanos de la ONU.

REDE (2013), Informe del relator especial sobre el derecho a la educación, A/68/294, Ginebra, Consejo de Derechos Humanos de la ONU. 
REDE (2012), Informe del relator especial sobre el derecho a la educación, Kishore Singh. Medidas normativas para una educación de calidad, A/HRC/20/21, Ginebra, Consejo de Derechos Humanos de la ONU.

Reglamento de la Junta de Gobierno de la Universidad Veracruzana (2014), aprobado en sesión ordinaria 103 de la H. Junta de Gobierno, celebrada el 9 de mayo, Xalapa, Universidad Veracruzana.

Suprema Corte de Justicia de la Nación (SCJN) (2017a), "Derecho a la educación superior. Su contenido y características", Semanario Fudicial de la Federación, 10a. Época, 1a. Sala, J. 83/2017 (10a.), México, 20 de octubre.

SCJN (2017b), "Universidades privadas. La negativa de aplicar a sus alumnos exámenes parciales y finales, cuando el derecho a presentarlos se encuente establecido en su normativa interna, es un acto de particular equivalente a los de autoridad, impugnable en el amparo indirecto", Semanario Judicial de la Federación, [TA], 10a. Época, T.C.C., XXVII.3o.33 A (10a.), 27 de octubre.

SCJN (2017c), "Derecho a la educación pública superior.

El Estado mexicano tiene la obligación de implantar progresivamente su gratuidad", Semanario Fudicial de la Federación, [J], 10a. Época, 1ª. Sala, 1a./J.84/2017 (10a.), 20 de octubre.

SCJN (2016), "Autonomía universitaria. constituye una garantía institucional del derecho a la educación superior, por lo que no puede ser utilizada para restringirlo", Gaceta del Semanario Fudicial de la Federación, [TA], 10a. Época, la. Sala, 1a. CCXCV/2016 (10a.), 2 diciembre.
SCJN (2014), "Sesiones públicas de los Tribunales Colegiados de Circuito. No son espacios de deliberación política sino procesos de debate jurídico entre magistrados cuyo objetivo final es la emisión de sentencias (interpretación de los artículos 184, 185, 186 y 187 de la Ley de Amparo", Gaceta del Semanario fudicial de la Federación, [TA], 10a. Época, 1a. Sala, CCXCV/2016 (10a.), 12 noviembre.

Serrano, Sandra y Daniel Vázquez (2014), Los derechos en acción. Obligaciones y principios de derechos humanos, México, Facultad Latinoamericana de Ciencias Sociales.

Tribunales Colegiados de Circuito (TCG) (2017a), "Universidades privadas. La negativa de aplicar a sus alumnos exámenes parciales yfinales, cuando elderecho a presentarlos se encuentra en su normativa interna, es un acto de particular equivalente a los de autoridad, impugnable en el amparo indirecto", [TA] 10a. Época, T.C.C., en Gaceta del Semanario Fudicial de la Federación, XXVII.3o.33 A (10a.), publicación 27 de octubre. TCG (2017b), "Universidades privadas. La obligación impuesta a sus alumnos de usar una pulsera, brazalete $\mathrm{u}$ otro distintivo análogo, para diferenciar quienes han pagado los servicios educativos que prestan, es un acto particular equivalente a los de autoridad, impugnables en el amparo indirecto", en Semanario Fudicial de la Federación, [TA], 10a. Época, T.C.C., XXVII.3o.32 A (10a.), 27 de octubre. Universidad Autónoma de Baja California (UABC) (2014), "Inicia proceso selección Rector UABC", Baja California, UABC, disponible en: <https://www. youtube.com/watch? $\mathrm{V}=$ gxwmtdklwly $>\quad$ [Consulta: diciembre de 2017]. 
UNESCO (2009), Conferencia Mundial sobre la Educación Superior: la nueva dinámica de la educación superior y la investigación para el cambio social y el desarrollo, París, UNESCO.

UNESCO (1998), Declaración mundial sobre la educación superior en el siglo XXI: visión y acción, París, UNESCO. UNESCO (1997), Recomendación relativa a la condición del personal docente de enseñanza superior, París, UNESCO.

UNESCO(1996), ConferenciaRegionalsobre Políticasyestrategias para la transformación de la educación superior en América Latina y el Caribe, La Habana-Cuba, UNESCO.
Vargas, Rosa y Emir Olivares (2007), "Arcaico, el proceso para designar al rector de la UNAM, afirma Ordorika", La fornada, 28 de septiembre, disponible en: <http:// www.jornada.unam.mx/2007/09/28/index.php? section $=$ sociedad $\&$ article $=048 \mathrm{n} 1 \mathrm{soc}>$ [Consulta: noviembre de 2017].

Vidal, Javier y María Vieira (2014), "Gobierno, autonomía y toma de decisiones en la Universidad", Revista de Pedagogía, vol. 66, núm. 1, pp. 17-30.

\section{Cómo citar este artículo:}

Jongitud-Zamora, Jaqueline-del-Carmen y Claudio-Rafael Castro-López (2019), "Las Juntas de Gobierno en el contexto del derecho a la educación superior. La experiencia de la Universidad Veracruzana en el proceso de designación rectoral 2017”, en Revista Iberoamericana de Educación Superior (RIES), México, UNAM-IISUE/Universia, vol. X, Núm. 29, pp. 195-213, DOI: https://doi.org/10.22201/ iisue.20072872e.2019.29.531 [consulta: fecha de última consulta]. 